\title{
Freeform Lens Design for Illumination with Different Luminance Intensities
}

Ku Chin Lin*

Mechanical Engineering Department, Kun Shan University, Taiwan

\begin{abstract}
Mapping of equi-luminous fluxes from a point source through a freeform lens for illumination with different luminance intensities is studied. The freeform surface is associated with the mapping and the grids to design on a target plane. Relocation of the target grids designed for uniform illumination is proposed by comparing the grids with reference and desired ones for interpolation to achieve the desired illumination distribution. Target-grids relocation for rectangular illumination with vertical, horizontal, tilt, circular, ring and rectangular cut-off for two different luminance intensities is demonstrated. The target grids after relocation are smooth in distribution, and the lens with a smooth freeform surface is achieved. The adequacy of the proposed methodology is proved by simulation.
\end{abstract}

Keywords: Luminance intensities; Target-grids

\section{Introduction}

A freeform lens has variety of applications such as LED TV, microprojector, auto fog light and headlight, etc. [1,2]. Methods for freeform lens design are primarily based on the grids mapped by equi-luminous flux rays on a target plane. Typical methods may include those to calculate global surface grids simultaneously [3] and local surface grids successively $[4,5]$. The surface grids are required to be smooth to ensure the applicability of the edge-ray principle [6,7] and the satisfaction of the integrability condition [8].

Freeform lens design for uniform illumination has been widely studied, but for illumination with different luminance intensities the studies are few. Lenses with discrete freeform surfaces may be designed to generate illumination with different luminance intensities [4] However, it could be inadequate in practice if the size of the source is a significant factor, and the manufacturing cost is still high currently $[9,10]$.

A pioneer work to design a freeform lens for illumination with two different luminance intensities was proposed [11]. Three alphabets "oec" with higher luminance intensity than the background is achieved using a tailored-made lens. However, an efficient approach to construct the grids mapped by equi-luminous flux rays was missing. It deserves an advanced study to make the design of a lens for illumination with two different luminance intensities become more efficient. Henceforth, initiation of such a study is proposed here.

In this study, a new approach to design the grids mapped by equiluminous flux rays is proposed. The grids to design are called the target grids (TG). Illumination grid (IG) is introduced over the illumination area. The IG is associated with the luminance intensity distribution. For example, it is uniformly distributed for uniform distribution of luminance intensity. Uniformly-distributed IG will be defined as reference illumination grids (RIG). The IG associated with desired luminance distribution with different intensities is named desired illumination grid (DIG). The proposed approach to calculate the TG for illumination with two different intensities is based on relocation of the TG for uniform illumination and interpolation of the DIG among the RIG.

\section{Target Grids for Uniform Illumination}

Typical candela distribution of an LED light is of a Lambertian type.
The distribution and its accumulation after normalization are given in Figure 1 . The accumulation is equally divided into $N_{\varphi}$ divisions along the inclination and $N_{\theta}$ divisions along the azimuthal,

$$
\begin{aligned}
& E_{\varphi}\left(\varphi_{p}^{*}\right)=\int_{0}^{\varphi_{s, i}^{*}} e\left(\varphi_{s}\right) d \varphi_{s}=\frac{i}{N_{\varphi}}, i=1,2, \ldots . ., N_{\varphi} \\
& E_{\theta}\left(\theta_{s, j}^{*}\right)=\int_{0}^{\theta_{s, j}^{*}} e\left(\theta_{s}\right) d \theta_{s}=\frac{j}{N_{\theta}}, j=1,2, \ldots . ., N_{\theta}
\end{aligned}
$$

where $\varphi_{s}$ denotes the inclination angle of a light ray; e the candela distribution; $\varphi_{s, i}^{*}$ the inclination angle of equi-luminous flux; $\theta_{s, j}^{*}$ the azimuthal angle of equi-luminous flux; $E_{\varphi}\left(\varphi_{s, i}^{*}\right)$ and $E_{\theta}\left(\theta_{s, j}^{*}\right)$ accumulation of equi-luminous flux. For uniform illumination, the TG denoted by the $\varphi_{p}^{*}$-contours and the $\varphi_{p}^{*}$-contours in Figure 2 are for illumination with circular, rectangular, triangular, and trapezoidal contours [12,13]. For non-circular illumination, variation of the contours is smooth from an inner ellipse toward the rectangular. The designed freeform lenses for the TG are in Figure 3. The outer contours of the lenses agree with the illumination contours. Simulation results of luminance intensity distribution generated by the lenses using Light
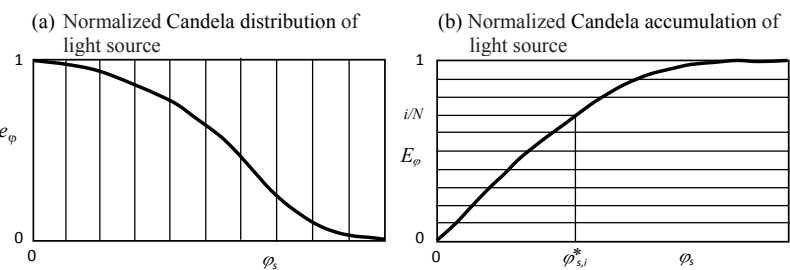

Figure 1: Normalized candela distribution and accumulation of light source. a. Normalized Candela distribution of light source. b. Normalized Candela accumulationoflight source.

*Corresponding author: Ku Chin Lin, Mechanical Engineering Department, Kun Shan University, Taiwan, Tel: 886-6-2057122; E-mail: kclin@mail.ksu.edu.tw

Received January 13, 2015; Accepted February 02, 2015; Published February 05, 2015

Citation: Lin KC (2015) Freeform Lens Design for Illumination with Different Luminance Intensities. J Comput Sci Syst Biol 8: 099-103. doi:10.4172/ jcsb. 1000175

Copyright: (c) 2015 Lin KC. This is an open-access article distributed under the terms of the Creative Commons Attribution License, which permits unrestricted use, distribution, and reproduction in any medium, provided the original author and source are credited. 
Citation: Lin KC (2015) Freeform Lens Design for Illumination with Different Luminance Intensities. J Comput Sci Syst Biol 8: 099-103. doi:10.4172/ jcsb. 1000175

(a) Target grids for circular contour of uniform illumination

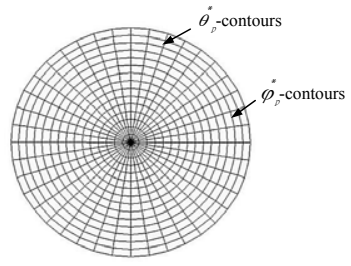

(c) Target grids for triangular contour of uniform illumination

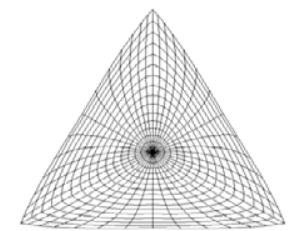

(b) Target grids for rectangular contour of uniform illumination

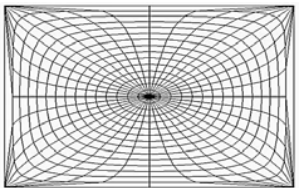

(d) Target grids for trapezoidal contour of uniform illumination

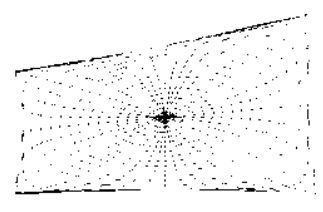

Figure 2: Target grids for design of freeform lenses for different contours of uniform illumination. a. Target grids for circular contour of uniform illumination. b. Target grids for rectangular contour of uniform illumination. c. Target grids for triangular contour of uniform illumination. d. Target grids for trapezoidal contour of uniform illumination.

(a) Lens for circular contour of uniform illumination

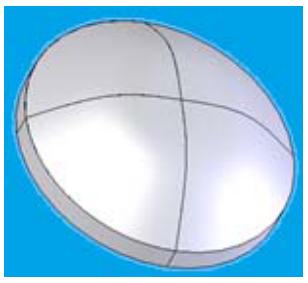

(c) Lens for triangular contour of uniform illumination

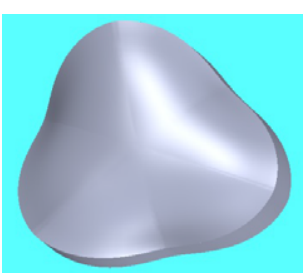

(b) Lens for rectangular contour of uniform illumination

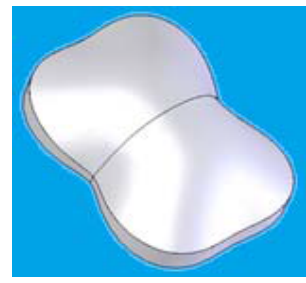

(d) Lens for trapezoidal contour of uniform illumination

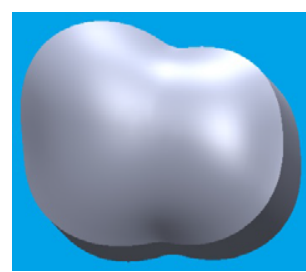

Figure 3: Freeform lenses designed based on the target grids shown in Figure 2 for different contours of uniform illumination. a. Lens for circular contour of uniform illumination. b. Lens for rectangular contour of uniform illumination. c. Lens for triangular contour of uniform illumination. d. Lens for trapezoidal contour of uniform illumination.

Tools for $N_{\phi}=50, N_{\theta}=200$ and 50,000 tracing rays are shown in Figure 4. The luminance intensity is normalized and good uniformity of illumination is achieved.

\section{Example Studies}

An example of RIG for rectangular uniform illumination is in Figure 5a. Then RIG is uniformly-s-distributed grids and described below:

$$
R_{x}(k)=\frac{k-1}{N_{x}-1} W, k=1, . ., N_{x}
$$

$$
R_{y}(k)=\frac{k-1}{N_{y}-1} H, k=1, . ., N_{y}
$$

where $W$ denotes the width of the rectangle; $H$ the height of the rectangle $\mathrm{R}_{\mathrm{x}}(k)$ and $\mathrm{R}_{\mathrm{y}}(\mathrm{k})$; the distance of $k^{\text {th }}$ grid from the left edge and the bottom edge of the rectangle, respectively; $N_{x}$ the number of grids distributed along the $x$ axis; $N$, the number of grids along the $y$ axis. The DIG for illumination with a vertical cut-off line at $x=0$ is shown in Figure 5b. The DIG for illumination with a horizontal cut-off line at $y=0$ is in Figure $5 c$. The DIG for illumination with a tilt cut-off (a) Luminance intensity distribution generated by the circular lens

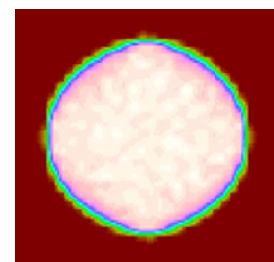

(c) Luminance intensity distribution generated by the triangular lens

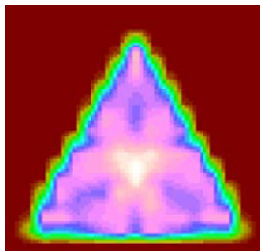

(b) Luminance intensity distribution generated by the rectangular lens

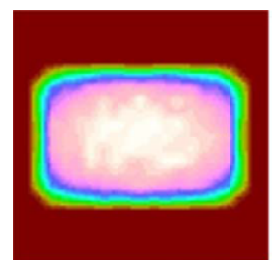

(d) Luminance intensity distribution generated by the trapezoidal lens

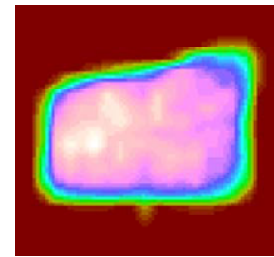

Figure 4: Simulated luminance intensity distribution generated by the lens shown in Figure 3 for different contours of uniform illumination. a. Luminance intensity distribution generated by the circular lens. b. Luminance intensity distribution generated by the rectangular lens. c. Luminance intensity distribution generated by the triangular lens. d. Luminance intensity distribution generated by the trapezoidal lens. (a) Reference illumination grids for rectangular illumination

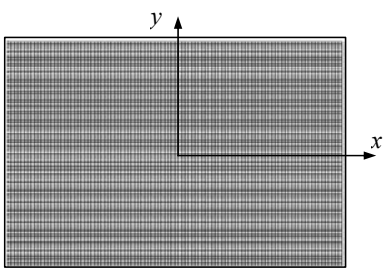

(c) Desired illumination grids for rectangular illumination with horizontal cut-off

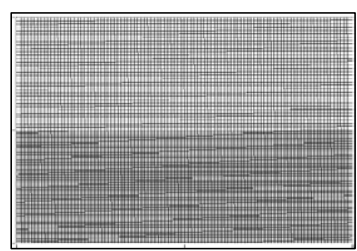

(b) Desired illumination grids for rectangular illumination with vertical cut-off

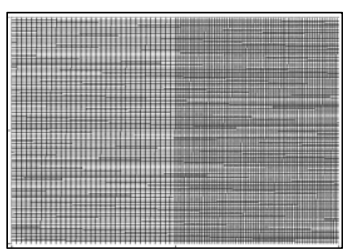

(d) Desired illumination grids for rectangular illumination with tilt cut-off

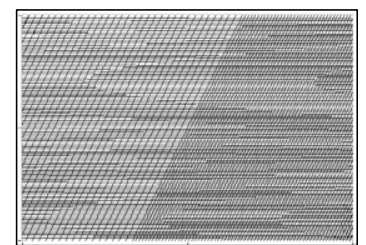

Figure 5: Reference and desired illumination grids for rectangular illumination with vertical, horizontal and tilt cut- off. a. Reference illumination grids for rectangular illumination. b. Desired illumination grids for rectangular illumination with vertical cut-off. c. Desired illumination grids for rectangular illumination with horizontal cut-off. d. Desired illumination grids for rectangular illumination with tilt cut-off. 
Citation: Lin KC (2015) Freeform Lens Design for Illumination with Different Luminance Intensities. J Comput Sci Syst Biol 8: 099-103. doi:10.4172/ jcsb. 1000175

line at $(x, y)=(0,0)$ is shown in Figure $5 \mathrm{~d}$ where the slope of the line is 2. The TG for rectangular uniform illumination is in Figure 6a. The distribution density at the right is twice higher than that at the left and it is described as below:

$$
D_{x}(k)=\left\{\begin{array}{c}
\left(\frac{k-1}{N_{x}-1}\right) \frac{3 W}{2}, k=1, . ., \frac{N_{x}-1}{3}+1 \\
\left(\frac{k-1}{N_{x}-1}-\frac{1}{3}\right) \frac{3 W}{2}, k=1, . ., \frac{N_{x}-1}{3}+2, \ldots, N_{x}
\end{array}\right.
$$

where $\mathrm{D}_{\mathrm{x}}(k)$ the distance of the $k^{\text {th }}$ grid from the left edge of the rectangle.

The distance of each of the TG from the left edge of the rectangle is given below:

$$
T_{d}(i, j)=T_{x}(i, j)+\frac{W}{2}, i=1, \ldots, N_{\varphi}, j=1, N_{\theta}
$$

where $\mathrm{T}_{x}(i, j)$ denotes the $x$ coordinate of the $(i, j)^{\text {th }}$ grid of the TG.

By comparing the $x$ coordinate of each of the TG with $\mathrm{R}_{x}(k)$, from $k=1$ until $\mathrm{R}_{x}\left(k^{*}\right)<\mathrm{T}_{d}(i, j) \leq \mathrm{R} x\left(k^{*}+1\right)$, and we have

$$
\begin{aligned}
& T_{x}^{\prime}(i, j)=D_{x}\left(k^{\prime}\right)-\frac{W}{2}, i=1, \ldots, N_{\varphi}, j=1, \ldots, N_{\theta} \\
& T_{y}^{\prime}(i, j)=T_{y}(i, j), i=1, \ldots, N_{\varphi}, j=1, \ldots, N_{\theta}
\end{aligned}
$$

Where

$$
k^{\prime}=k^{*}+\frac{T_{d}(i, j)-R_{x}\left(k^{*}\right)}{R_{x}\left(k^{*}+1\right)-R_{x}\left(k^{*}\right)}, i=1, \ldots, N_{\varphi}, j=1, \ldots, N_{\theta}
$$

and $\mathrm{T}_{y}(i, j)$ denotes the $y$ coordinate of the $(i, j)^{\text {th }}$ grid of the TG; $\left(T_{x}^{\prime}, T_{y}^{\prime}\right)$ denotes the TG after interpolation and it is in Figure 6b. The DIG for illumination with a horizontal cut-off line at $y=0$ is in Figure $5 \mathrm{c}$. The distribution density at the bottom is twice higher than that at the top, and it is described as below: (a) Target grids for rectangular contour of uniform illumination

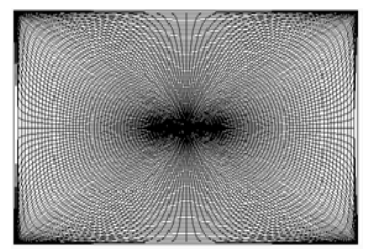

(c) Target grids for rectangular illumination with horizontal cut-off

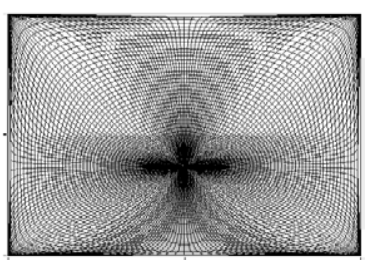

(b) Target gridsfor rectangular illumination with vertical cut-off

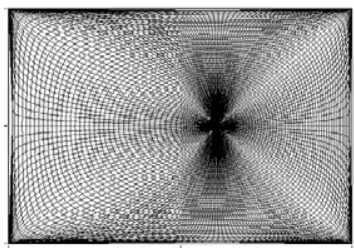

(d) Target gridsfor rectangular illumination with tilt cut-off

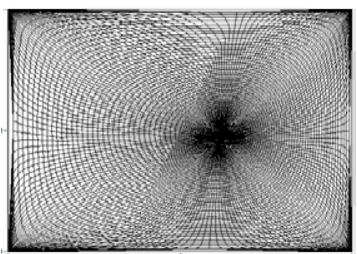

Figure 6: Target grids for rectangular illumination with vertical, horizontal and tilt cut-off. a. Target grids for rectangular contour of uniform illumination. b. Target grids for rectangular illumination with vertical cut-off. c. Target grids for rectangular illumination with horizontal cut-off. d. Target grids for rectangular illumination with tilt cut-off.

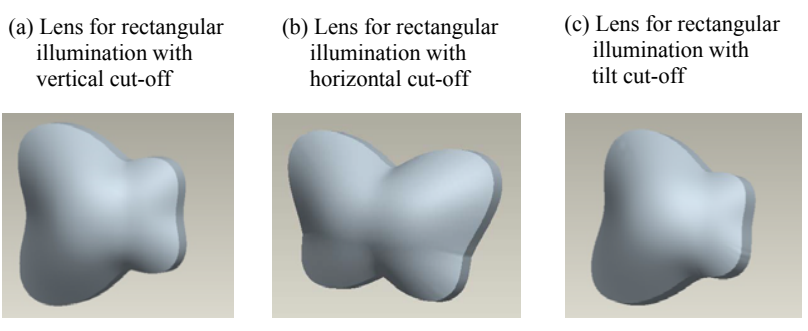

Figure 7: Freeform lenses designed based on the target grids shown in Figure 6 for rectangular, illumination with vertical, horizontal and tilt cut-off. a. Lens for rectangular illumination with vertical cut-off. b. Lens for rectangular illumination with horizontal cut-off. c. Lens for rectangularillumination with tilt cut-off.

$$
D_{y}(k)=\left\{\begin{array}{c}
\left(\frac{k-1}{N_{y}-1}\right) \frac{3 W}{2}, k=1, . ., \frac{N_{y}-1}{3}+1 \\
\left(\frac{k-1}{N_{y}-1}-\frac{1}{3}\right) \frac{3 W}{2}, k=1, . ., \frac{N_{y}-1}{3}+2, \ldots, N_{y}
\end{array}\right.
$$

where $D_{y}(k)$ the distance of the $k^{\text {th }}$ grid from the bottom edge of the rectangle.

The distance of the TG from the bottom edge of the rectangle is given below:

$$
T_{d}(i, j)=T_{y}(i, j)+\frac{W}{2}, i=1, . ., N_{\varphi}, j=1, N_{\theta}
$$

where $\mathrm{T}_{\mathrm{y}}(i, j)$ denotes the y coordinate of the $(i, j)^{\text {th }}$ grid of the TG.

By comparing the $y$ coordinate of each of the TG with $\mathrm{R} y(k)$, from $k=1$ until $\mathrm{R} y\left(k^{*}\right)<\mathrm{T}_{d}(i, j) \leq \mathrm{R} y\left(k^{*}+1\right)$, and we have

$$
\begin{aligned}
& T_{y}^{\prime}(i, j)=D_{y}\left(k^{\prime}\right)-\frac{H}{2}, i=1, \ldots, N_{\varphi}, j=1, \ldots, N_{\theta} \\
& T_{x}^{\prime}(i, j)=T_{x}(i, j), i=1, \ldots, N_{\varphi}, j=1, \ldots, N_{\theta}
\end{aligned}
$$

Where

$$
k^{\prime}=k^{*}+\frac{T_{d}(i, j)-R_{y}\left(k^{*}\right)}{R_{y}\left(k^{*}+1\right)-R_{y}\left(k^{*}\right)}, i=1, \ldots, N_{\varphi}, j=1, \ldots, N_{\theta}
$$

and $\mathrm{T}_{x}(i, j)$ denotes the $\mathrm{x}$ coordinate of the $(i, j)^{\mathrm{th}}$ grid of TG; the TG after interpolation is shown in Figure $6 \mathrm{c}$.

The TG after interpolation is shown in Figure 6d. The DIG for illumination with a tilt cut-off line at $(x, y)=(0,0)$ is shown in Figure $5 \mathrm{~d}$ where the slope of the line is 2 . The designed freeform lenses for the above DIG are shown in Figure 7. The outer contours of the lenses agree with the unbalanced illumination. Simulation results of 50,000 tracing rays using Light Tools for $N_{\phi}=50$ and $\mathrm{N}_{\theta}=200$ are shown in Figure 8, and the desired illumination with two different luminance intensity is achieved. For illumination with a circular, ring and rectangular cutoff area, the TG after relocation is shown in Figure 9. The designed freeform lenses are in Figure 10. The freeform surfaces of the lenses are popped-up with a smooth circular, ring and rectangular area. Simulation results of luminance intensity distribution generated by the lenses are in Figure 11, and illumination with desired distribution of luminance intensity is achieved. 
Citation: Lin KC (2015) Freeform Lens Design for Illumination with Different Luminance Intensities. J Comput Sci Syst Biol 8: 099-103. doi:10.4172/ jcsb. 1000175

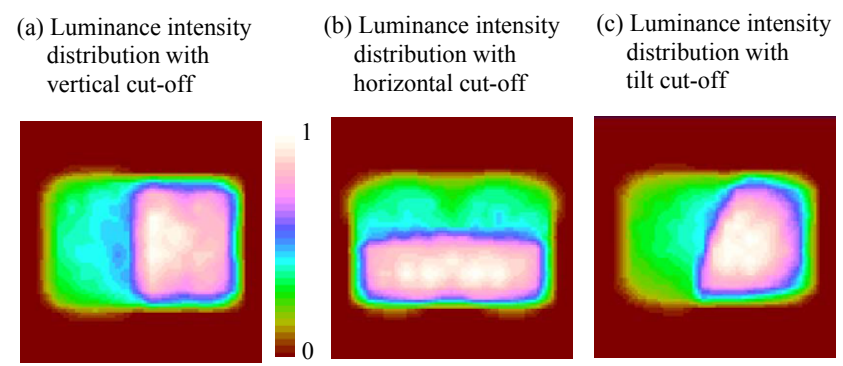

Figure 8: Simulated luminance intensity distributions generated by the lenses shown in Figure 7 for rectangular illumination with vertical, horizontal and tilt cut-off. a. Luminance intensity distribution with vertical cut-off. b. Luminance intensity distribution with horizontal cut-off. c. Luminance intensity distribution with tilt cut-off.

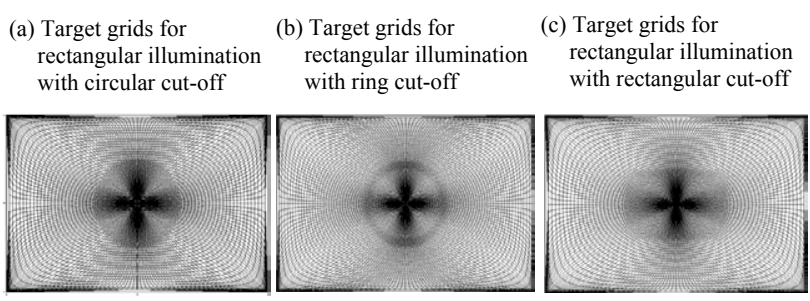

Figure 9: Target grids for rectangular illumination with circular, ring and rectangular cut-off contours. a. Target grids for rectangular illuminationrectangular with circular cut-off. b. Target grids for rectangular illumination with ring cut-off. c. Target grids for rectangular illumination with rectangular cut-off.

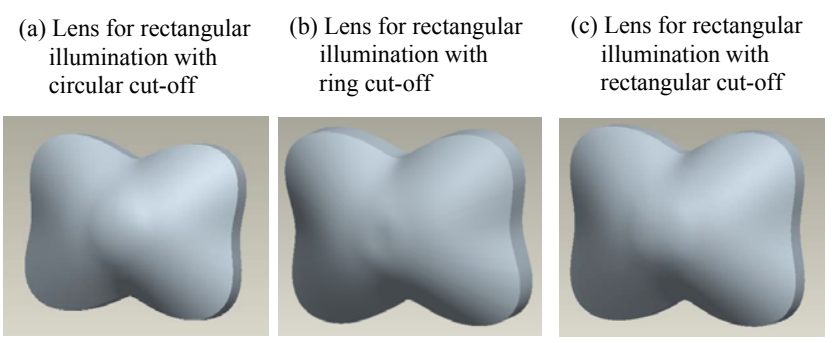

Figure 10: Freeform lenses designed based on the target grids shown in Figure 9 for rectangular illumination with circular, ring and rectangular cut-off. a. Lens for rectangular illumination with circular cut-off. b. Lens for rectangular illumination with ring cut-off. c. Lens for rectangular illumination with rectangular cut-off.

\section{Conclusion}

Freeform lens design for illumination with different luminance intensities is studied. The proposed approach is an extension of the previous one for uniform illumination. Examples of the TG of equiluminous fluxes for uniform illumination are given in Figure 2. The IG distribution is associated with the distribution of luminance intensity. Examples of RIG and DIG are given in Figure 5. Methods to relocate the TG for illumination with different luminance intensities are proposed by relocating the TG for uniform illumination and interpolation of the DIG among the RIG. The TG for rectangular illumination with vertical, horizontal, tilt, circular, ring and rectangular cut-off has been demonstrated in Figures 6 and 9 . The $\varphi_{p}^{*}$ and $\varphi_{p}^{*}$ contours vary smoothly

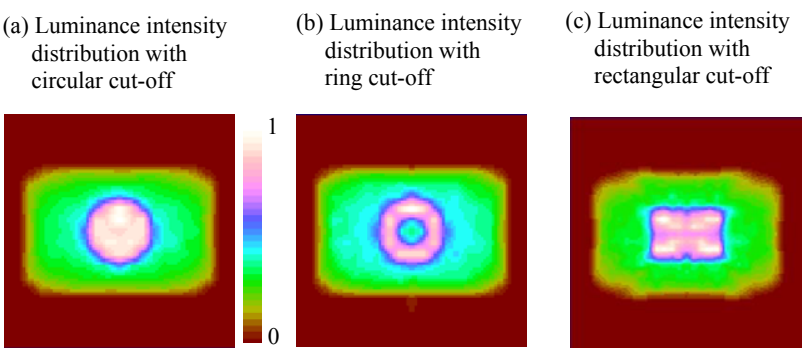

Figure 11: Simulated luminance intensity distribution generated by the lenses shown inFigure 10 for rectangular illumination with circular, ring and rectangular cut-off. a. Luminance intensity distribution with circular cut-off. b. Luminance intensity distribution with ring cut-off. c. Luminance intensity distribution with rectangular cut-off.

according to the interpolation. The freeform lenses designed by the proposed method are smooth as shown in Figures 7 and 10. The desired illumination is achieved as shown in Figures 8 and 11. This proves the adequacy of the proposed methodology. Extension for complicated and biased shapes of cut-off is currently under investigation.

\section{Acknowledgement}

This work was supported by the National Science Council, Taiwan, R.O.C under the grant NSC-103-2221-E-168-017-

\section{References}

1. Koonin EV, Mushegian AR, Galperin MY, Walker DR (1997) Comparison of archaeal and bacterial genomes: computer analysis of protein sequences predicts novel functions and suggests a chimeric origin for the archaea. Molecular Microbiology 25: 619-637.

2. Iwabe N, Kuma K, Hasegawa M, Osawa S, Miyata T (1989) Evolutionary relationship of archaebacteria, eubacteria, and eukaryotes inferred from phylogenetic trees of duplicated genes. Proceedings of the National Academy of Sciences 86: 9355-9359.

3. Woese CR, Fox GE (1977) Phylogenetic structure of the prokaryotic domain the primary kingdoms. Proc Natl Acad Sci USA 74: 5088-5090.

4. Woese CR, Achenbach L, Rouviere P, Mandelco L (1991) Archaeal phylogeny: reexamination of the phylogenetic position of Archaeoglobus fulgidus in light of certain composition-induced artifacts. Syst Appl Microbiol 14: 364-371.

5. van de Vossenberg JL, Driessen AJ, Konings WN (1998) The essence of being extremophilic: the role of the unique archaeal membrane lipids. Extremophiles 2: $163-170$.

6. Brochier-Armanet C, Boussau B, Gribaldo S, Forterre P (2008) Mesophilic Crenarchaeota: proposal for a third archaeal phylum, the Thaumarchaeota. Nat Rev Microbiol 6: 245-252.

7. Bult CJ, White O, Olsen GJ, Zhou L, Fleischmann RD, et al. (1996) Complete genome sequence of the methanogenic archaeon, Methanococcus jannaschii. Science 273: 1058-1073

8. Schneider KL, Pollard KS, Baertsch R, Pohl A, Lowe TM (2006) The UCSC Archaeal Genome Browser. Nucleic Acids Res 34: D407-410.

9. Roten CA, Gamba P, Barblan JL, Karamata D (2002) Comparative Genometrics (CG): a database dedicated to biometric comparisons of whole genomes. Nucleic Acids Res 30: 142-144

10. Mojica F, Ferrer C, Juez G, Rodriguez-Valera F (1995) Long stretches of short tandem repeats are present in the largest replicons of the Archaea Haloferax mediterranei and Haloferax volcanii and could be involved in replicon partitioning. Molecular microbiology 17: 85-93.

11. Capaldi SA, Berger JM (2004) Biochemical characterization of Cdc6/Orc1 binding to the replication origin of the euryarchaeon Methanothermobacter thermoautotrophicus. Nucleic Acids Res 32: 4821-4832.

12. Norais C, Hawkins M, Hartman AL, Eisen JA, Myllykallio H, et al. (2007) 
Citation: Lin KC (2015) Freeform Lens Design for Illumination with Different Luminance Intensities. J Comput Sci Syst Biol 8: 099-103. doi:10.4172/ jcsb. 1000175

Genetic and physical mapping of DNA replication origins in Haloferax volcanii. PLoS Genet 3: e77.

13. Zhang R, Zhang CT (2005) Identification of replication origins in archaeal genomes based on the Z-curve method. Archaea 1: 335-346.

14. Wu Z, Liu H, Liu J, Liu X, Xiang H (2012) Diversity and evolution of multiple orc/cdc6-adjacent replication origins in haloarchaea. BMC Genomics 13: 478.

15. Dueber EL, Corn JE, Bell SD, Berger JM (2007) Replication origin recognition and deformation by a heterodimeric archaeal Orc1 complex. Science 317 : 1210-1213.

16. Robinson NP, Bell SD (2005) Origins of DNA replication in the three domains of life. FEBS J 272: 3757-3766.

17. Coker JA, DasSarma P, Capes M, Wallace T, McGarrity K, et al. (2009) Multiple replication origins of Halobacterium sp. strain NRC-1: properties of the conserved orc7-dependent oriC1. J Bacteriol 191: 5253-5261.
18. Robinson NP, Dionne I, Lundgren M, Marsh VL, Bernander R, et al. (2004) Identification of two origins of replication in the single chromosome of the archaeon Sulfolobus solfataricus. Cell 116: 25-38.

19. Lopez $P$, Philippe H, Myllykallio H, Forterre $P$ (1999) Identification of putative chromosomal origins of replication in Archaea. Mol Microbiol 32: 883-886.

20. Pelve EA, Lindås AC, Knöppel A, Mira A, Bernander R (2012) Four chromosome replication origins in the archaeon Pyrobaculum calidifontis. Mol Microbiol 85 986-995.

21. Gao F, Zhang CT (2007) DoriC: a database of oriC regions in bacterial genomes. Bioinformatics 23: 1866-1867.

22. Gao F, Luo H, Zhang CT (2013) DoriC 5.0: an updated database of oriC regions in both bacterial and archaeal genomes. Nucleic Acids Res 41: D90-93.

23. Luo H, Zhang CT, Gao F (2014) Ori-Finder 2, an integrated tool to predict replication origins in the archaeal genomes. Front Microbiol 5: 482. 